\title{
Quantification of the surface stress in microcantilever biosensors: revisiting Stoney's equation
}

\author{
Javier Tamayo* ${ }^{*^{\dagger}}$, Jose J. Ruz*, Valerio Pini*, Priscila Kosaka and Montserrat Calleja \\ Institute of Microelectronics of Madrid, CSIC, Isaac Newton 8 (PTM), Tres Cantos, 28760 Madrid, Spain \\ Microcantilever biosensors in the static operation mode translate molecular recognition into a surface \\ stress signal. Surface stress is derived from the nanomechanical cantilever bending by applying Stoney's \\ equation, derived more than one hundred years ago. This equation ignores the clamping effect on the \\ cantilever deformation, which induces significant errors in the quantification of the biosensing response. \\ This leads to discrepancies in the surface-stress induced by biomolecular interactions in measurements \\ with cantilevers with different sizes and geometries. So far, more accurate solutions have been \\ precluded by the formidable complexity of the theoretical problem that involves solving the two- \\ dimensional biharmonic equation. In this Letter, we present an accurate and simple analytical \\ expression to quantify the response of microcantilever biosensors. The equation exhibits an excellent \\ agreement with finite elements simulations and experiments of DNA immobilization on gold-coated \\ microcantilevers.
}

*These three authors contributed equally to this work.

+Corresponding author: jtamayo@imm.cnm.csic.es

Phone: +34 918063338 
More than one hundred years ago, George Gerald Stoney studied the peeling of metallic films deposited electrolytically on glass, a phenomenon that was detrimental in many industrial processes at that time. He observed "that metals are deposited under tension, and they should strain the material on which they were deposited so as to bend it, and that by the amount of this bending the tension could be determined"[1]. The relation derived to quantify the film tension from the plate bending, so called Stoney's equation, today is a cornerstone to quantify the surface stress and to characterize many relevant processes in different fields including adsorbate/surface interactions[2,3], surface reconstruction[4] and thin film deposition in microfabrication processes[5,6,7]. Molecular recognition on a biosensing surface also brings about a change of the surface stress that can be used as a fingerprint for biological detection[8]. This is the key concept of microcantilever biosensors operating in the static mode. One of the microcantilever surfaces is sensitized with molecular receptors that exhibit high binding affinity to the targeted analyte. When the cantilever is exposed to the sample solution, molecular recognition between the analyte and the surface-grafted receptors induces a stress difference between opposite cantilever sides that bend the cantilever structure $[9,10,11,12,13,14,15]$. Stoney's formula is used to derive the molecular recognition induced surface stress from the change in the microcantilever curvature.

Let us consider a cantilever with length $L$, width $b$, and thickness $h$, under uniform and isotropic strain-independent surface stress loading $\sigma$ on the upper face (Fig. 1). Stoney's model predicts that the cantilever bends with uniform curvature $(\kappa)$ in proportion to the surface stress following $[5,7,16]$,

$\kappa=\frac{h-h_{n}}{D(1+v)} \sigma$

where $v$ is the Poisson's ratio, $h_{n}$ is the distance between the neutral axis and the bottom face $(\mathrm{h} / 2$ for single material cantilevers) and $D$ is the biaxial flexural rigidity per unit width that for a single material cantilever is given by $\frac{E h^{3}}{12\left(1-v^{2}\right)}$ where E is the Young's modulus. Equation (1) assumes that the beam is isotropic and homogeneous, the beam thickness is small compared to the lateral dimensions, and the strains and rotations are small (the effect of in-plane stress on the out-of-plane displacement is negligible). This adequately complies with the most commonly used microcantilevers with a length, width and thickness that typically fall in the range 100-500 $\mu \mathrm{m}, 10$ $100 \mu \mathrm{m}, 0.1-1 \mu \mathrm{m}$, respectively. However, Stoney's equation is only strictly valid for plates unrestrained along the edges. Microcantilevers are rigidly clamped to a chip substrate and hence exhibit null displacement at its clamped end, which is not compatible with the uniform curvature 
predicted by Stoney[16] (Fig. 1). John E. Sader demonstrated that this effect can induce significant errors in the surface stress measurements and derived complex analytical solutions for the asymptotic cases $L>>b$ and $L<<b$ [16,17]. Interestingly, the clamping effect on the cantilever deformation is key to unveil a long standing unsolved problem in physics, the effect of surface stress on the resonance frequency of cantilevers[18,19]. A solution for the clamping effect on the deformation of a cantilever poses a formidable challenge and a simple and analytical solution is not tractable. In practice, simulations based on the finite element method must be performed to obtain accurate solutions as shown in the simulation in Fig. 1(b) for a cantilever with aspect ratio $L / b=2$ $[20,21]$.

In the present Letter, we derive a simple analytical formula for the deformation of a microcantilever averaged across the width as a function of the longitudinal coordinate that enables accurate quantification of the surface stress. The formula notably fits finite element simulations and experimental data.

By applying the principle of virtual displacements to the strain energy of a bent plate, we obtain the differential equation that governs the out of plane displacement $w(x, y)$ of a plate[22],

$\frac{\partial^{4} \mathrm{w}(\mathrm{x}, \mathrm{y})}{\partial \mathrm{x}^{4}}+\frac{\partial^{4} \mathrm{w}(\mathrm{x}, \mathrm{y})}{\partial \mathrm{y}^{4}}+2 \frac{\partial^{4} \mathrm{w}(\mathrm{x}, \mathrm{y})}{\partial \mathrm{x}^{2} \partial \mathrm{y}^{2}}=0$

and the boundary conditions at the free edges, $y= \pm b / 2$ and $x=L[16,22]$,

$\left\{\frac{\partial^{2} \mathrm{w}(\mathrm{x}, \mathrm{y})}{\partial \mathrm{n}^{2}}+v \frac{\partial^{2} \mathrm{w}(\mathrm{x}, \mathrm{y})}{\partial s^{2}}\right\}_{\text {free edge }}=\frac{\sigma\left(h-h_{n}\right)}{\mathrm{D}}$
$\left\{\frac{\partial^{3} \mathrm{w}(\mathrm{x}, \mathrm{y})}{\partial \mathrm{n}^{3}}+(2-v) \frac{\partial^{3} \mathrm{w}(\mathrm{x}, \mathrm{y})}{\partial \mathrm{n} \partial s^{2}}\right\}_{\text {free edge }}=0$

where $n$ and $s$ denote the normal and tangential coordinates at the cantilever boundary. Equations (3a) and (3b) state that at all free edges, the normal components of the bending moment and transverse shearing forces must be zero, respectively. To these boundary conditions, we must add the conditions that arise from the clamping restriction, i.e., no displacement and no rotation about the yaxis,

$\mathrm{w}(\mathrm{x}=0, \mathrm{y})=\left[\frac{\partial \mathrm{w}(\mathrm{x}, \mathrm{y})}{\partial \mathrm{x}}\right]_{\mathrm{x}=0}=0$

The mathematical equations (2)-(4) belong to a family of problems referred to as the biharmonic problem in a rectangle, a long standing benchmark problem in mathematics and engineering since 1811 [23]. The most accurate approximation for solving this problem, referred to as the 
superposition method, consists of constructing the solution in the form of the sum of two ordinary Fourier series in $x$ and $y$ [23]. However the achievement of the solution requires of solving an infinite series of linear equations, which in some cases suffers from low convergence. Recently, a similar approach has been adopted to achieve the surface-stress induced cantilever deformation[24]. However, the corresponding infinite coefficient matrix is ill-conditioned, which suggests both that the mathematical problem has a non exact solution, and the physical description of the problem is incorrect. Let us use a simple argument to demonstrate this conclusion. If we combine the bending moment boundary condition (eq. (3a)) and the clamping restriction (eq. (4)), we obtain that the displacement at the clamping corners must satisfy,

$v\left[\frac{\partial^{2} \mathrm{w}(\mathrm{x}, \mathrm{y})}{\partial \mathrm{s}^{2}}\right]_{\left(\mathrm{x}=0, \mathrm{y}= \pm \frac{\mathrm{b}}{2}\right)}=\frac{\sigma\left(h-h_{n}\right)}{\mathrm{D}}$

Clearly, this corner point condition is violated when Poisson's ratio is zero. The corner pathology indicates that the boundary conditions, eq. (3a) and (4) are not compatible. We find this discordance physically intuitive as the clamping constriction exerts a force distribution to counterbalance the curvature along the y-axis induced by the bending moment (Fig. 1(b)). Thus, it seems logical that the reaction force exerted by the clamped edge induces an effective bending moment near the clamping corners.

Given the complexity of the problem, we propose to relax one of the conflicting boundary conditions and to calculate the cantilever deflection averaged across the cantilever width. Firstly, we expand the solution as an infinite power series in $y$, in which only the even powers are retained due to the symmetry about the $\mathrm{x}$-axis ${ }^{23}$,

$$
w(\zeta, \eta)=\frac{1}{2} \kappa_{S t} b^{2}\left(\lambda^{2} \zeta^{2}+\eta^{2}\right)+\sum_{k=0}^{\infty} f_{k}(\zeta) \eta^{2 k}
$$

where $\zeta=x / L$ and $\eta=y / b$ are dimensionless coordinates used for the sake of physical significance, $\lambda=L / b$ is the aspect ratio of the cantilever and $\kappa_{S t}$ is the Stoney's curvature (eq. (1)). The first summand in eq. (6) is the Stoney's solution only valid for an unrestrained plate and the second summand is the effect of the clamping. Substitution of the proposed solution in the biharmonic equation (eq. (2)) gives the following relation,

$f_{k}(\zeta)=\frac{(-1)^{k-1}}{(2 k) ! \lambda^{2 k}}\left[(k-1) \frac{d^{2 k} f_{0}(\zeta)}{d \zeta^{2 k}}+2 k \lambda^{2} \frac{d^{2 k} f_{1}(\zeta)}{d \zeta^{2 k}}\right]$

The biharmonic equation reduces the number of functions involved in the solution, from infinity independent functions to two independent functions, $\mathrm{f}_{0}$ and $\mathrm{f}_{1}$. In the $\mathrm{x}$-direction, the clamping induced strain must decay from its maximum value near the clamp region to its nominal 
(Stoney) value remote from the clamping. The question is what kind of decaying functions to use. In beam mechanics, it has been found that singularities such as cracks or boundary restrictions give rise to exponentially decaying functions and this is also assumed for the present problem $[18,19,25]$. We find that exponential functions with the same exponent, $f_{0}(\zeta)=A_{0} e^{-\alpha \lambda \zeta}$ and $f_{1}(\zeta)=A_{1} e^{-\alpha \lambda \zeta}$, are the only functions that satisfy the boundary conditions imposed below. Substituting these proposed functions in equation (6), we obtain,

$$
w(\zeta, \eta)=\frac{1}{2} \kappa_{S t} b^{2}\left(\lambda^{2} \zeta^{2}+\eta^{2}\right)+e^{-\alpha \lambda \zeta}\left\{\mathrm{A}_{0} \operatorname{Cos}(\alpha \eta)+\frac{\left(2 \mathrm{~A}_{1}+\mathrm{A}_{0} \alpha^{2}\right)}{2 \alpha} \eta \operatorname{Sin}(\alpha \eta)\right\}
$$

Now we apply the lateral edge boundary conditions (eqns. 3(a) and 3(b)) and a relaxed clamping condition: the integral of the displacement and slope in y along the clamping must be zero. The satisfaction of these boundary conditions provides a very simple analytical expression for the deflection averaged over y:

$\bar{w}(\zeta) \equiv \int_{-\frac{1}{2}}^{\frac{1}{2}} d \eta w(\zeta, \eta)=\frac{1}{2} \kappa_{S t} b^{2} \lambda^{2} \zeta^{2}+\kappa_{S t}\left(\frac{\mathrm{b}}{\alpha}\right)^{2} v\left(e^{-\alpha \lambda \zeta}+\alpha \lambda \zeta-1\right)$

where the exponent $\alpha$ only depends on the Poisson's ratio and it satisfies the equation,

$\alpha(1-v)+(3+v) \operatorname{Sin}(\alpha)=0$

The solution of equation (10) was numerically obtained and it was fitted to a second order polynomial in the Poisson's ratio,

$\alpha(v) \cong 2.2803+1.0056 v-0.1762 v^{2}$

The obtained solution indicates that the clamping restriction induces an additional curvature to the uniform curvature derived by Stoney. This term exponentially decays in the $x$ direction with a characteristic length scale that is linearly proportional to the cantilever's width with a proportionality constant that weakly varies from 0.44 to 0.37 when the Poisson's ratio is increased from 0 to 0.5 . The clamping-induced curvature gives a relative increase in the free end deflection with respect to the Stoney's value of $\approx \frac{2 v}{\alpha \lambda}$ when $\lambda>>1$. Figure 2 shows the longitudinal curvature averaged over the cantilever's width obtained from our analytical solution (line) and finite element method (FEM) simulations (symbols) for a cantilever with a short aspect ratio $(\lambda=2)$ and several values of Poisson's ratio (Fig. 2(a)) and for a typical Poisson's ratio of 0.25 and several values of aspect ratio (Fig. 2(b)). The values of the curvature are normalized by the Stoney's curvature. The FEM simulations consisted on a quadrilateral mapped mesh of $\approx 50 \mathrm{k}$ solid elements with a $99.9 \%$ convergence in the deflection at the free cantilever end. The analytical solution (eqns. (9) and (11)) and FEM results 
exhibit a remarkable agreement. The discrepancy between the two solutions becomes significant for the extreme case of aspect ratio $\lambda=1$, in which the deviation between the curvatures at the cantilever free end is of about $2.5 \%$. However, even in this case the deflection at the free end only shows a discrepancy of $0.05 \%$. Given the accuracy of our formula, we apply equations (9) and (11) to assess the overestimation of the surface stress obtained when the Stoney's formula is used in typical experiments where the cantilever deflection is measured near the free end by optical methods (Fig. 3). The results show that the error becomes significant as the aspect ratio of the cantilever is reduced and for materials with high Poisson's ratio. The error can largely increase when electrical transduction techniques based on piezoresistivity or capacitive measurements are used, as the output voltage is proportional to the strain near the clamping or to the average deflection, respectively.

The simplicity of the derived equations is highlighted when we derive the curvatures in $x$ and $y$ averaged over the cantilever's width that are respectively given by,

$$
\begin{aligned}
& \bar{\kappa}_{x}(x) \equiv \int_{-\frac{b}{2}}^{\frac{b}{2}} d y \frac{\partial^{2} w(x, y)}{\partial \mathrm{x}^{2}}=\kappa_{S t}\left(1+v e^{-\alpha(v) \frac{x}{b}}\right) \\
& \bar{\kappa}_{y}(x) \equiv \int_{-\frac{b}{2}}^{\frac{b}{2}} d y \frac{\partial^{2} w(x, y)}{\partial \mathrm{y}^{2}}=\kappa_{S t}\left(1-e^{-\alpha(v) \frac{x}{b}}\right)
\end{aligned}
$$

Notice that we have, in this case, recovered the normal coordinates $x$ and $y$ to emphasize the simplicity of the obtained solutions. Appealingly, the combination of equations 12(a) and 12(b) provide a useful and simple relationship,

$$
\bar{\kappa}_{x}(x)+v \bar{\kappa}_{y}(x)=\kappa_{S t}(1+v) .
$$

that means that the longitudinal bending moment averaged over the cantilever's width is equal to the surface stress induced bending moment for all $\mathrm{x}$.

Figure 4 shows the comparison between the averaged curvatures in $\mathrm{x}$ and $\mathrm{y}$ obtained by equations (12a) and (12b), by FEM simulation and by the analytical approximation of Sader for cantilevers with high aspect ratio that is the reference model [16]. We have chosen a cantilever with low aspect ratio $\lambda=2$ and other with typical aspect ratio of $\lambda=5$ as the cantilever used here in the experiments (see below). The results indicate that (i) the simple equations derived here (equations (12a) and (12b)), accurately follows the FEM simulations, and (ii) the more complex solution derived by Sader[16] shows significant deviation near the clamping.

Finally, we have performed experiments to validate our analytical expression for the surface stress. We used silicon cantilevers $500 \mu \mathrm{m}$ long, $100 \mu \mathrm{m}$ wide and $1 \mu \mathrm{m}$ thick (Concentris). The cantilevers were coated by sputtering evaporation with a $20 \mathrm{~nm}$ gold layer on top of a $2 \mathrm{~nm}$ adhesion 
layer of chromium. Freshly coated microcantilevers were incubated with $1 \mu \mathrm{M}$ of a thiolated ssDNA probe (5'-HS- CTACCTTTTTTTTCTG-3') diluted in phosphate buffered saline (PBS) at pH 7.4 for 24 hours in order to immobilize a densely packed DNA layer [26]. Afterwards, the cantilevers were rinsed with PBS buffer and Milli- $Q^{\circledR}$ water to discard unspecific interactions and dried under dry nitrogen. The nanometer-scale out-of-plane displacements of the cantilevers were measured in air at a relative humidity of $40 \%$ and room temperature by scanning laser beam deflection microscopy $[27,28]$. Figure 5 shows the average deflection variation induced by the chemisorption of the ssDNA molecules. Since the effect of the clamping exponentially decays with a characteristic length $\approx \mathrm{b} / 2.5$, we assume that the curvature in the last third part of the cantilever is uniform and follows Stoney's equation. Thus we fitted this region to a second order polynomial (Fig. 5(a)) to discriminate the clamping effect from the difference between the displacement profile and the fitting curve. The obtained difference curve, plotted in Fig. 5(b), has been fitted to the exponential function predicted in equations (9) and (11) (line). The experimental and theoretical curves exhibit a remarkable resemblance. The only fitting parameter was the effective Poisson's ratio that is 0.08 . This value is in the range of the expected values taking into account the uncertainty in the thickness of the cantilever and gold layer. Poisson's ratio for silicon and gold approximately are 0.064 and 0.44 , respectively [29].

In conclusion, we have revisited the problem of the surface stress measurement by the cantilever bending method. This problem has gained relevance in the last decade after the emergence of nanomechanical sensors. Surprisingly, the advances in micro- nanofabrication of cantilevers, transduction techniques and biomedical applications have not been accompanied by the development of more sophisticated methods to quantify the cantilever response, in this case the surface stress. To date, most of the works in this field still use the Stoney's formula presented in 1909 in the Proceeding of the Royal Society A[1]. Stoney's equation ignores the effect of the clamping restriction[16,17]. However, a more accurate formula has been precluded due to the formidable complexity of the biharmonic problem in a rectangle, a still challenging problem in mathematics and engineering [23]. In addition to this difficulty, we find that the boundary conditions classically proposed are incorrect as they ignore the reaction force exerted by the clamp. We circumvent all these difficulties by restricting the theory to find cantilever deflection averaged across the cantilever width and by relaxing one of the conflicting boundary conditions, in this case the clamping restriction. We obtain that the clamping effect on the cantilever deformation can be simply described as an exponentially decaying term in the $x$ direction with a characteristic length scale that is linearly proportional to the cantilever's width and weakly depends on Poisson's ratio. The new formula will enable an accurate surface stress determination removing the discrepancy between experiments due to the use of cantilevers with different geometries. In addition, these measurements can thus be compared with measurements obtained with other techniques such as contact angle[8] or X-ray diffraction[30]. 
The authors acknowledge financial support from the Spanish Science Ministry through projects ANEM! (TEC2009-14517-C02) and INMUNO-swing (IPT-2011-0821-010000); and from European Research Council through starting grant NANOFORCELLS (ERC-StG-2011-278860). The authors acknowledge fruitful discussion with A. San Paulo.

\section{REFERENCES}

1. Stoney GG (1909) The tension of metallic films deposited by electrolysis. Proceedings of the Royal Society of London Series A, Containing Papers of a Mathematical and Physical Character 82: 172-175.

2. Ibach H (2009) Adsorbate induced surface stress. Journal of Vacuum Science \& Technology A: Vacuum, Surfaces, and Films 12: 2240-2245.

3. Haiss W (2001) Surface stress of clean and adsorbate-covered solids. Reports on Progress in Physics 64: 591.

4. Ibach H (1997) The role of surface stress in reconstruction, epitaxial growth and stabilization of mesoscopic structures. Surface science reports 29: 195-263.

5. Freund L, Floro J, Chason E (1999) Extensions of the Stoney formula for substrate curvature to configurations with thin substrates or large deformations. Applied Physics Letters 74: 1987.

6. Freund LB, Suresh S (2003) Thin film materials: stress, defect formation, and surface evolution: Cambridge Univ Pr.

7. Hsueh CH (2002) Modeling of elastic deformation of multilayers due to residual stresses and external bending. Journal of Applied Physics 91: 9652.

8. Bergese P, Oliviero G, Colombo I, Depero LE (2009) Molecular recognition by contact angle: proof of concept with DNA hybridization. Langmuir 25: 4271-4273.

9. Fritz J, Baller M, Lang $H$, Rothuizen $H$, Vettiger $P$, et al. (2000) Translating biomolecular recognition into nanomechanics. Science 288: 316.

10. Raiteri R, Grattarola M, Butt HJ, Skládal P (2001) Micromechanical cantilever-based biosensors. Sensors and Actuators B: Chemical 79: 115-126.

11. Wu G, Datar RH, Hansen KM, Thundat T, Cote RJ, et al. (2001) Bioassay of prostate-specific antigen (PSA) using microcantilevers. Nature biotechnology 19: 856-860.

12. Arlett J, Myers E, Roukes M (2011) Comparative advantages of mechanical biosensors. Nature Nanotechnology 6: 203-215.

13. Boisen A, Thundat T (2009) Design \& fabrication of cantilever array biosensors. Materials Today 12: 32-38.

14. Fritz J (2008) Cantilever biosensors. Analyst 133: 855-863.

15. Waggoner PS, Craighead HG (2007) Micro-and nanomechanical sensors for environmental, chemical, and biological detection. Lab Chip 7: 1238-1255.

16. Sader JE (2001) Surface stress induced deflections of cantilever plates with applications to the atomic force microscope: Rectangular plates. Journal of Applied Physics 89: 2911.

17. Sader JE (2002) Surface stress induced deflections of cantilever plates with applications to the atomic force microscope: V-shaped plates. Journal of Applied Physics 91: 9354.

18. Lachut MJ, Sader JE (2007) Effect of surface stress on the stiffness of cantilever plates. Physical review letters 99: 206102. 
19. Lachut MJ, Sader JE (2012) Effect of surface stress on the stiffness of thin elastic plates and beams. Physical Review B 85: 085440.

20. Dahmen K, Lehwald S, Ibach H (2000) Bending of crystalline plates under the influence of surface stress-a finite element analysis. Surface science 446: 161-173.

21. Ricci A, Ricciardi C (2010) A new Finite Element approach for studying the effect of surface stress on microstructures. Sensors and Actuators A: Physical 159: 141-148.

22. Timoshenko S, Woinowsky-Krieger S, Woinowsky S (1959) Theory of plates and shells: McGraw-hill New York.

23. Meleshko V (1997) Biharmonic problem in a rectangle. Applied scientific research 58: 217249.

24. Zeng X, Deng J, Luo X (2012) Deflection of a cantilever rectangular plate induced by surface stress with applications to surface stress measurement. Journal of Applied Physics 111: 083531-083531-083538.

25. Christides S, Barr A (1984) One-dimensional theory of cracked Bernoulli-Euler beams. International Journal of Mechanical Sciences 26: 639-648.

26. Mertens J, Rogero C, Calleja M, Ramos D, Martín-Gago JA, et al. (2008) Label-free detection of DNA hybridization based on hydration-induced tension in nucleic acid films. Nature nanotechnology 3: 301-307.

27. Martínez N, Kosaka P, Tamayo J, Ramírez J, Ahumada O, et al. (2010) High throughput optical readout of dense arrays of nanomechanical systems for sensing applications. Review of Scientific Instruments 81: 125109-125109-125109.

28. Tamayo J, Pini V, Kosaka P, Martinez NF, Ahumada O, et al. (2012) Imaging the surface stress and vibration modes of a microcantilever by laser beam deflection microscopy. Nanotechnology 23: 315501.

29. Hopcroft MA, Nix WD, Kenny TW (2010) What is the Young's Modulus of Silicon? Microelectromechanical Systems, Journal of 19: 229-238.

30. Xiong G, Huang X, Harder R, Robinson IK (2011) Differential stress induced by thiol adsorption on facetted nanocrystals. 


\section{FIGURES}

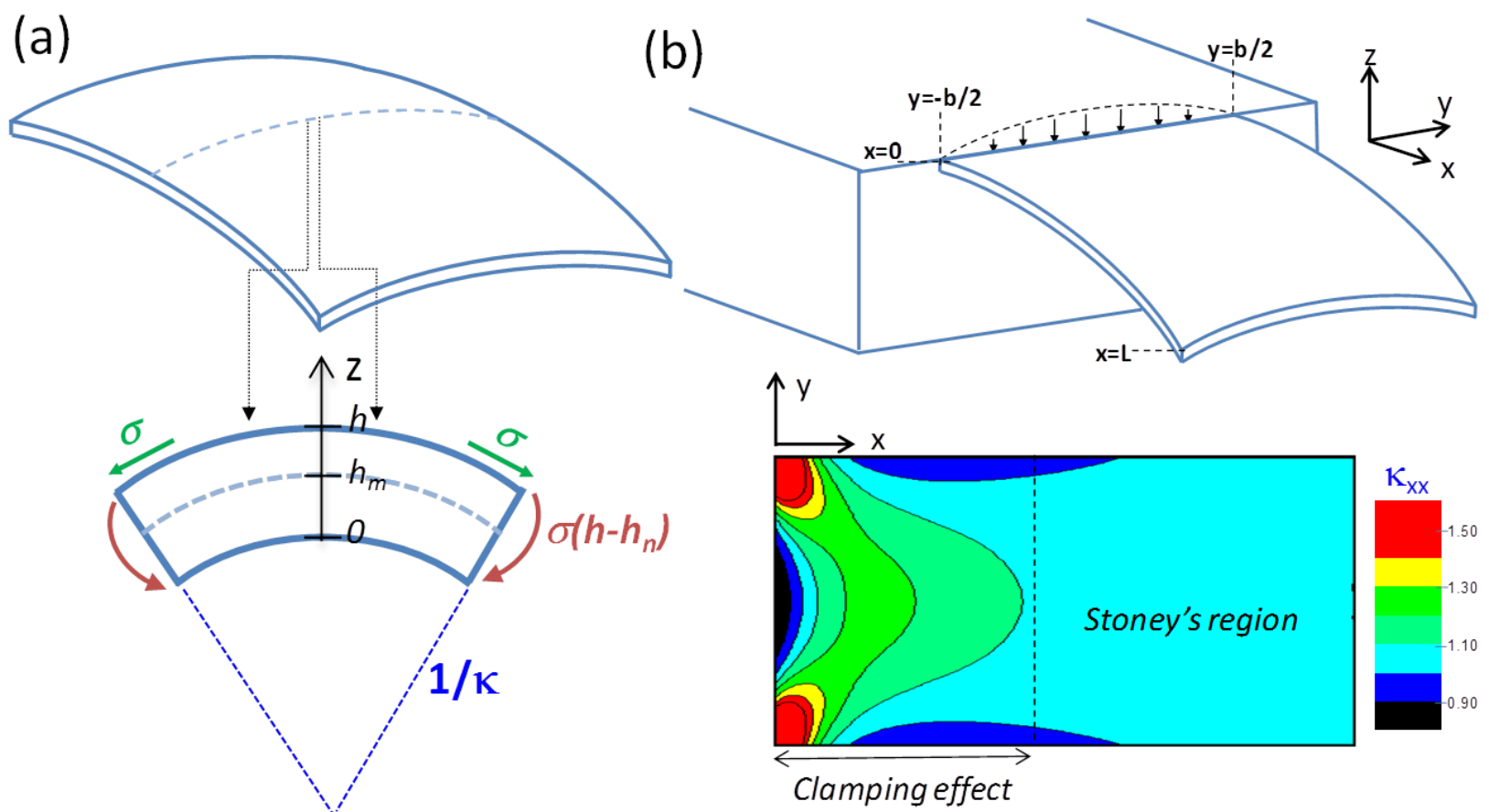

FIG. 1. (a) Schematic of the bending of an unrestrained rectangular plate when surface stress is developed in the upper face. The bottom diagram shows the surface forces, $\sigma$, and bending moments, $\sigma\left(h-h_{m}\right)$ exerted on a cross-section element of the plate. The plate bends with uniform curvature $\kappa$. (b) Schematic of the bending of the rectangular plate shown in (a) when it is clamped to a substrate. The coordinate system used in this work is also shown. The clamping exerts reaction forces to cancel out the curvature induced by the bending moment. This results into a non uniform curvature as shown in the finite element simulations of the curvature in $x\left(\kappa_{x x}\right)$ shown in the bottom for a cantilever with an aspect ratio $L / b=2$. The simulations show that the clamping induces a curvature that decays in the $x$ direction with a characteristic length scale given by the cantilever width $b$. The cantilever region where $x>b$ approximately exhibits a uniform curvature that obeys Stoney's equation. 


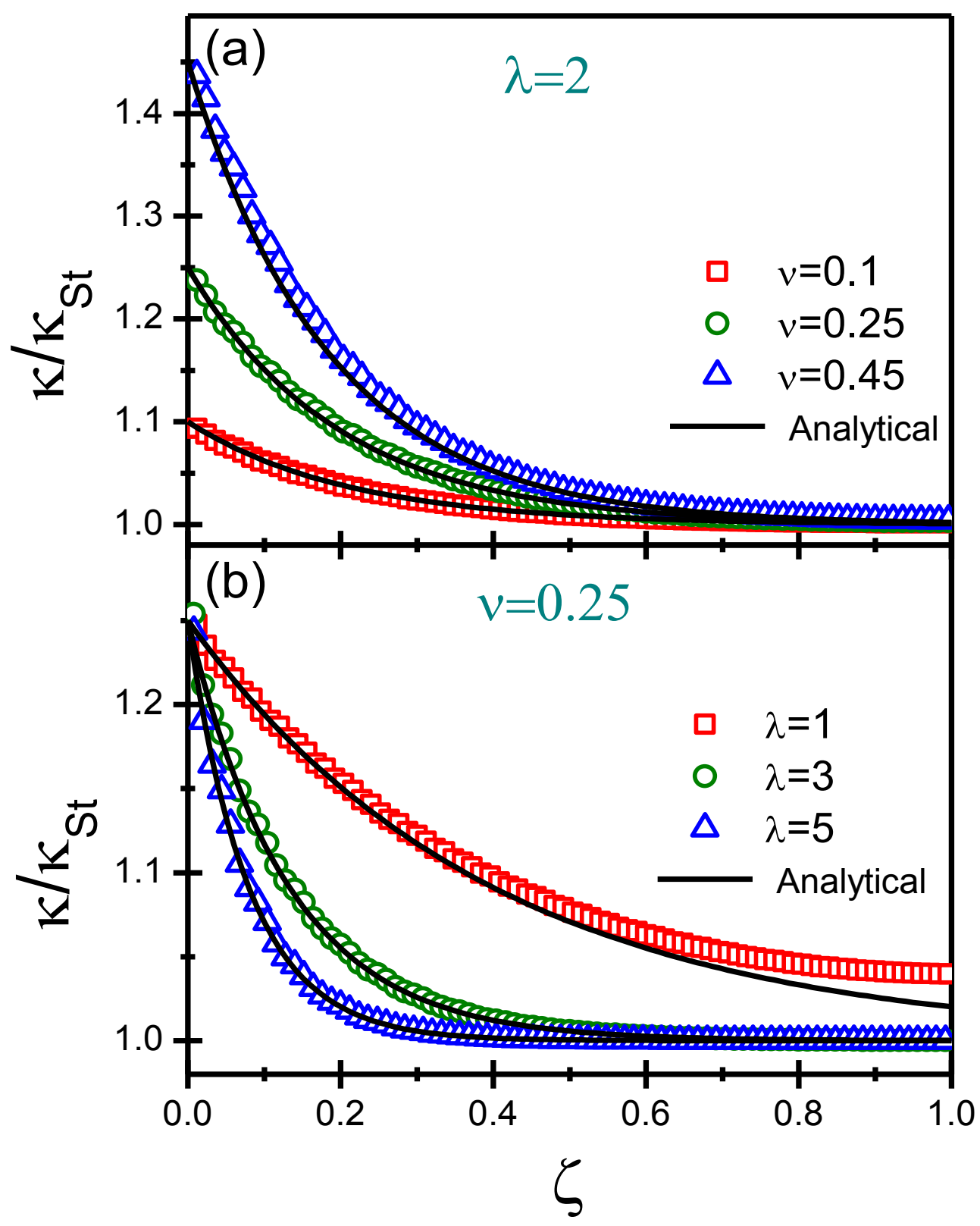

FIG. 2. Cantilever curvature in $\mathrm{x}$ averaged over $\mathrm{y}$ and normalized to the Stoney's curvature vs. $\zeta=\mathrm{x} / \mathrm{L}$ obtained by finite element simulations (symbols) and by the improved formula presented here (line) (see text). The comparison is performed for a fixed aspect ratio $\lambda=\mathrm{L} / \mathrm{b}=2$ and three values of the Poisson's ratio, $0.1,0.25$ and 0.45 (a) and for a fixed Poisson's ratio of 0.25 and three values of the aspect ratio, 1, 3 and 5 . 


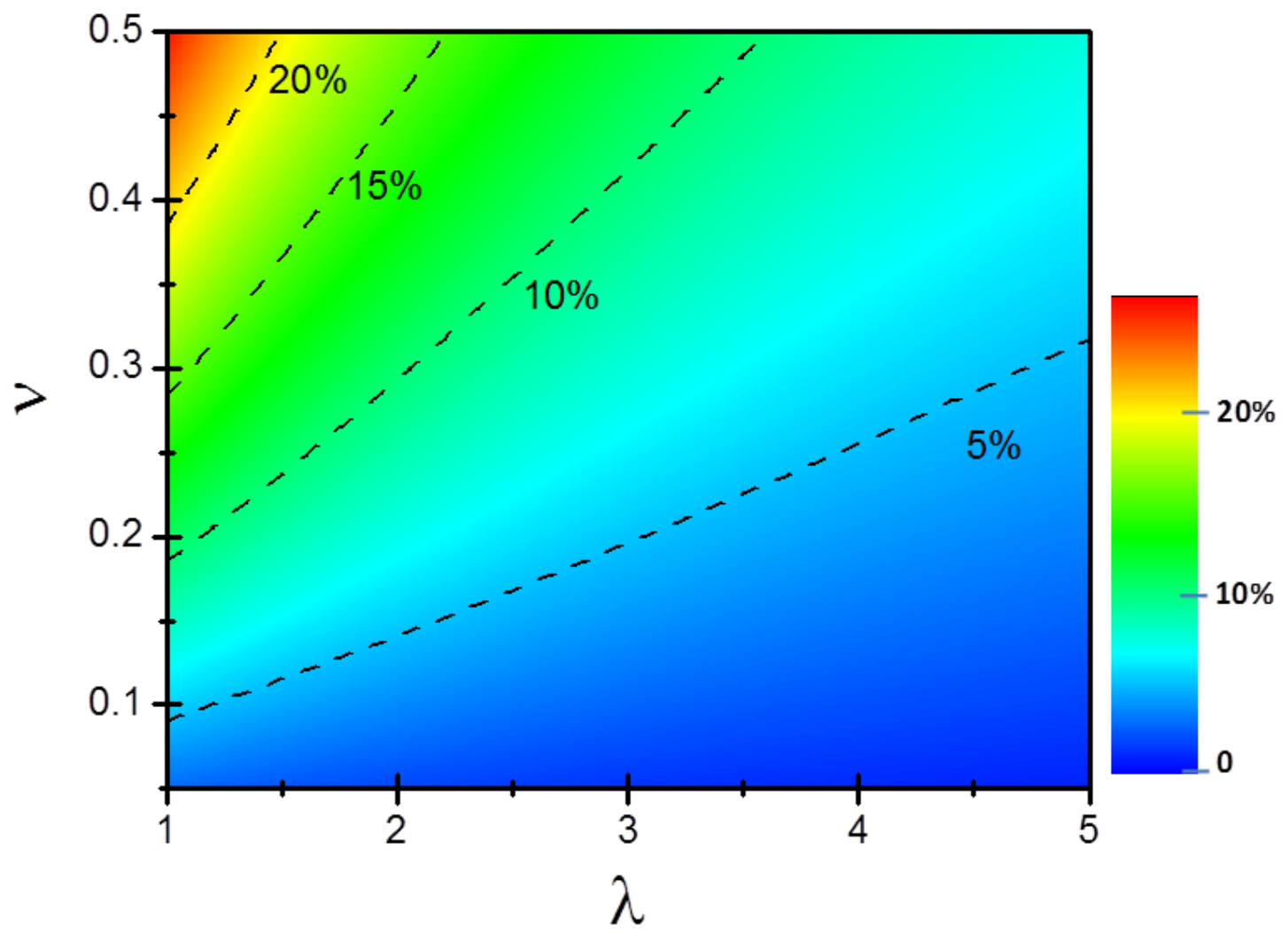

FIG. 3. Color intensity map of the overestimation of the surface stress obtained by applying the Stoney's equation in typical measurements of the cantilever deflection near the free end $(x=0.9 \mathrm{~L})$, as a function of the cantilever aspect ratio and Poisson's ratio of the cantilever material. 


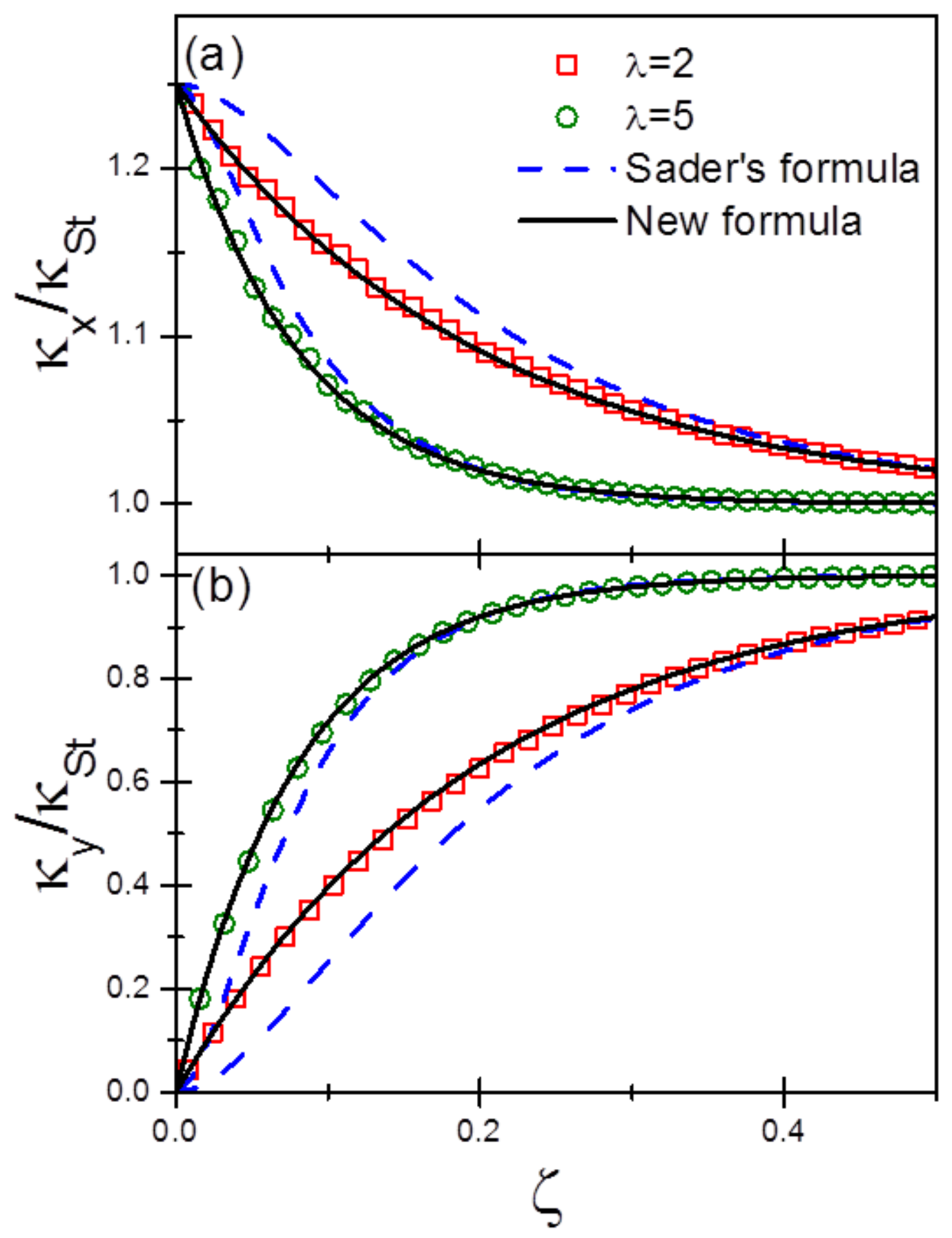

FIG. 4 Curvatures in x (a) and y (b) averaged over y and normalized to the Stoney's curvature as a function of $\zeta=\mathrm{x} / \mathrm{L}$ obtained by FEM simulations (symbols), by Sader's approximation (dashed blue line) and by the formulae presented here (black continuous line). The comparison is performed for a Poisson's ratio of 0.25 and two values of the aspect ratio $(\lambda), 2$ and 5 . The curves were plotted between $\zeta=0$ and $\zeta=0.5$ to highlight the effect of the clamping on the curvatures. For $\zeta>1 / \lambda$ the curvatures asymptotically reach the Stoney's curvature. 


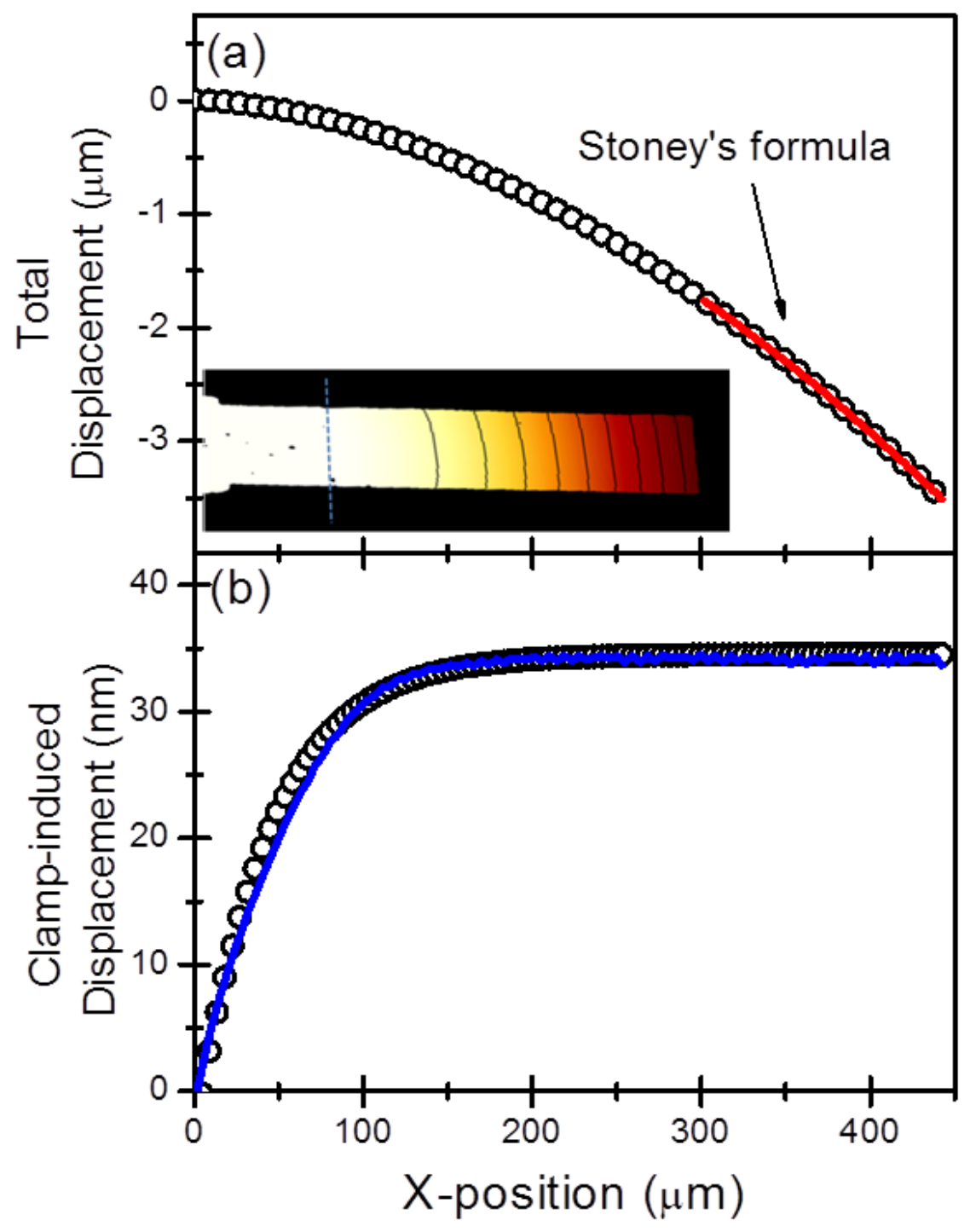

FIG. 5. (a) Variation of the mean cantilever deflection of a gold-coated silicon cantilever upon chemisorption of thiolated single stranded DNA (symbols). The cantilever is $500 \mu \mathrm{m}$ long, $100 \mu \mathrm{m}$ wide and approximately 1 $\mu \mathrm{m}$ thick. The measurements were obtained by scanning laser beam deflection microscopy. An image of the cantilever obtained by this technique is shown in the inset. The region near the free end is fitted to a second order polynomial (red line) as this region approximately follows Stoney's equation. The resulting parabola is subtracted to the total cantilever deflection to obtain the clamping induced deformation shown in (b) as symbols. The experimental data shown in (b) is fitted to the analytical formula presented in the main text (line). The experimental data is obtained from the average of ten measurements of the same cantilever. The error is much smaller than the symbol size. 